\title{
ERYTHROMYCIN INDUCED CARBAMAZEPINE TOXICITY- A PREVENTABLE DRUG INTERACTION
}

\author{
D Shrestha ${ }^{1 *}$, AK Dhakal ${ }^{1}, K_{K}$ Singh ${ }^{1}$ \\ ${ }^{1}$ Department of Pediatrics, KIST Medical College, Imadol, Lalitpur, Nepal. \\ *Correspondence to : Dr Devendra Shrestha, Department of Pediatrics, KIST Medical College, Imadol, Lalitpur, Nepal. \\ Email:neetag@hotmail.com
}

\begin{abstract}
A drug interaction between Erythromycin and Carbamazepine is well established and harmful effects of Carbamazepine toxicity can be avoided. We report a patient who developed features of Carbamazepine toxicity namely ataxia, vomiting and nystagmus within 24 hours of oral Erythromycin with high serum Carbamazepine level and improved after discontinuation of Erythromycin with returning of serum Carbamazepine level to therapeutic range. Awareness among health professionals, patient education, proper record keeping and strengthening pharmacy services are essential to prevent such drug interactions.
\end{abstract}

Key Words: Carbamazepine toxicity, Drug toxicity, Erythromycin-Carbamazepine interaction.

\section{INTRODUCTION}

Erythromycin is well known to cause increased levels of Carbamazepine when administered simultaneously and lead to Carbamazepine toxicity. The purpose of presenting this report is to highlight the importance of drug interaction between these commonly prescribed medications which can be avoided.

\section{Case report}

A 13 year-old male child diagnosed with idiopathic epilepsy was on oral Carbamazepine at a dose of $15 \mathrm{mg} / \mathrm{kg} /$ day and had remained seizure free for last 1.5 years. He presented to our OPD with complaints of dizziness, headache, vomiting, blurring of vision and unsteady gait for one day. There was no history of fever, seizure, loss of consciousness, urinary incontinence and head injury. There was no history of taking extra doses of Carbamazepine. On further inquiry, he was being prescribed oral Erythromycin at a dose of $50 \mathrm{mg} / \mathrm{kg} /$ day in divided doses for atypical pneumonia for last two days. There was history of similar complaints one year ago which occurred after mistakenly taking double doses of Carbamazepine and subsided after adjusting the proper doses. He was afebrile, conscious and well oriented with GCS 15/15. His pulse was 64/min regular and normal volume, BP $100 / 70 \mathrm{mmHg}$ and respiratory rate was $24 /$ min. Fundus was on normal on ophthalmoscopy. He had nystagmus in both eyes, ataxic gait, however coordination, disdiadochokinesia and tremor were absent. Meningeal signs were absent and rest of neurological examination was normal. Investigations revealed: Hemoglobin 12gm/dl, TLC 3700/ mm3, N43 L50 M3 E4, Platelets 346000/mm3, serum Na+ $132 \mathrm{mmol} / \mathrm{L}$, serum $\mathrm{K}+4.9 \mathrm{mmol} / 1$, serum urea $18.7 \mathrm{mg} /$ dl, serum creatinine $0.7 \mathrm{mg} / \mathrm{dl}$, blood sugar $88 \mathrm{mg} / \mathrm{dl}$, SGPT $37 \mathrm{U} / \mathrm{L}$, SGOT $25 \mathrm{U} / \mathrm{L}$, serum bilirubin $0.27 \mathrm{mg} / \mathrm{dl}$, Alkaline phosphatase 128U/L and serum Carbamazepine level $>18 \mathrm{mcg} /$ $\mathrm{ml}$ (Therapeutic range 4-10 mcg/ml). ECG and Peripheral smear was normal. Erythromycin induced Carbamazepine toxicity was considered and he was admitted in Pediatric ward and started on antiemetics and maintenance intravenous fluid. Erythromycin was stopped and Carbamazepine dose was withheld for one day and restarted on next day with reduced dose of $10 \mathrm{mg} / \mathrm{kg} /$ day. Neuroimaging and lumbar puncture for CSF analysis was planned but his symptoms improved remarkably on supportive therapy, so it was withheld after discussion with parents and patient was discharged after 48 hours. He was asymptomatic at first follow up, with serum Carbamazepine level of $7.6 \mathrm{mcg} / \mathrm{ml}$ and now under regular follow up with no break through seizure and ataxia.

\section{DISCUSSION}

Carbamazepine is commonly used antiepileptic agent in children with generalized and partial seizures. Carbamazepine is metabolized by cytochrome P-450 enzymes in liver and Carbamazepine ${ }^{10,11}$ epoxide is principal metabolite which has antiepileptic and neurotoxic property similar to Carbamazepine. Erythromycin is frequently used macrolide antibiotic in Penicillin allergic children and atypical pneumonia. Pharmacokinetically Erythromycin causes decreased metabolism of carbamazepine by inhibiting cytochrome P-450 CYP3A4 enzyme. ${ }^{1}$

Infection may itself depress CYP activity and along with Erythromycin induced CYP 3A4 inhibition may be responsible for increased Carbamazepine levels. ${ }^{1}$ Genetic factors may be responsible for difference in activity of individual P-450 and hence difference in susceptibility for drug interaction in 
different individuals. ${ }^{1}$ Our patient may also be genetically more susceptible to Carbamazepine as he had developed features of toxicity earlier when he had mistakenly taken double doses of Carbamazepine. The concurrent pneumonia and also co-administration of Erythromycin and probable increased susceptibility of Carbamazepine may be responsible for features of Carbamazepine toxicity in our patient.

Symptoms of Carbamazepine toxicity usually appear within 24 hours of starting therapy with Erythromycin and resolves rapidly within 48 to 72 hours after discontinuation of either drug. ${ }^{2,3}$ Similar clinical scenario was observed in this patient also.

Nystagmus, ataxia, drowsiness, sinus tachycardia are most common features observed in children with carbamazepine toxicity.4 Other features include neurological (agitation, coma, seizure, status epilepticus, respiratory depression, apnea, chorea, hypertonia, diplopia), cardiovascular (atrioventricular block, loss of $\mathrm{p}$ wave, widened QRS complex, hypotension, ventricular tachycardia), vomiting, ileus, hyponatremia, and hypokalemia. ${ }^{5}$ Carbamazepine toxicity results various clinical features in a dose dependent manner. Serum levels of Carbamazepine $>12 \mathrm{mg} / 1(50 \mathrm{mmol} / \mathrm{l})$ are associated with ataxia and nystagmus, and levels $>40 \mathrm{mg} / 1(170 \mathrm{mmol} / \mathrm{l})$ are associated with coma, respiratory depression and seizures. ${ }^{5,6}$

Patients receiving antiepileptic medications are prone to develop significant drug interactions even in developed countries. $^{7}$ Problem may arise due to failure to take complete history by health professionals or withholding information or prescriptions by patients. Patient may be taking treatment from different health professionals at same time in Nepal. Parents of children on long term treatment with Carbamazepine should be well educated about importance of disclosing their drug information to treating health professionals and also about possible interaction of Carbamazepine with other drugs. Special cards or badges with written information of receiving treatment with Carbamazepine should be carried by patients. Written instructions regarding avoiding specific drugs which cause interaction with Carbamazepine may also be helpful. ${ }^{2}$ Medical professionals also should be cautious while prescribing drugs with potential drug interactions and the value of obtaining detailed history cannot be undermined. Pharmacists also have important role to prevent this type of incident by reviewing prescriptions while dispensing medicines. However limited availability of these services in Nepalese context is another factor for drug interaction. Lastly, if Erythromycin has to be given in a child receiving Carbamazepine, the dose of Carbamazepine must be reduced and patient should be closely monitored during the period.

In conclusion, Erythromycin is commonly used antimicrobial agent in children and have propensity for drug interaction. Health care professionals have to be cautious when other drugs are concurrently used with Erythromycin. Proper drug history, review of past treatment history, knowledge of potential drugdrug interaction among health professionals and electronic prescribing will help to reduce similar drug interaction in future. Other congeners of macrolide can be used instead of
Erythromycin in patients receiving Carbamazepine to prevent such untoward event.

The author(s) declare that they have no competing interests. The author(s) received no financial support for the research and/or authorship of this article.

\section{REFERENCES}

1. Westphal JF. Macrolide-induced clinically relevant drug interactions with cytochrome P-450A (CYP) A4: an update focused on clarithromycin, azithromycin and dirithromycin. Br J Clin Pharmacol 2000;50:285-95.

2. Loganathan N, Sujithkumar S, Bhuvaneswari K, Thiagarajan VK. Acute Carbamazepine toxicity: A case of rare drug interaction. Indian Journal of Pharmacy Practice 2012;5(2):59-60.

3. Hedrick R, Williams F, Morin R, Lamb WA, Cate JC. Carbamazepine-erythromycin interaction leading to carbamazepine toxicity in four epileptic children. Ther Drug Monit 1983;5(4):405-7.

4. Lifshitz M, Gavrilov V, Sofer S. Signs and symptoms of carbamazepine overdose in young children. Pediatr Emerg Care. 2000;16(1):26-7.

5. Spiller HA. Management of carbamazepine overdose. Pediatr Emerg Care 2001; 17(6):452-6.

6. Hojer J, Malmlund $\mathrm{HO}$, Berg A. Clinical features in 28 consecutive cases of laboratory confirmed massive poisoning with carbamazepine alone. J Toxicol Clin Toxicol 1993; 31(3):449-58.

7. Novak PH, Ekins-Daukes S, Simpson CR, Milne RM, Helms P, McLay JS. Acute drug prescribing to children on chronic antiepilepsy therapy and the potential for adverse drug interactions in primary care. $\mathrm{Br} \mathrm{J}$ Clin Pharmacol 2004;59(6):712-7. 